\title{
Actividades profesionales confiables para psiquiatría.
}

\section{Entrustable professional activities for psychiatry.}

\author{
Silvana Sarabia ${ }^{1, a ; 2,2, b, c, d}$
}

En los últimos años el modelo basado por competencias que realiza una planificación "hacia atrás", define al egresado exitoso o competente y sus atributos, identifica la forma en que los graduados adquirirán esos atributos, crea un programa que incluye cómo serán evaluadas las competencias; definitivamente revolucionó la manera de enseñar y aprender medicina en pre y posgrado (1). En los programas por competencias, lo que se espera que el alumno adquiera al finalizar su entrenamiento, se especifica en términos de objetivos de aprendizaje o competencias. Sin embargo, otra manera de expresar el producto final es identificar las actividades que se le puede confiar al estudiante competente con la finalidad de vincular el concepto de competencia con la práctica clínica. Mientras que una competencia describe el conocimiento, la habilidad y la actitud que un estudiante debe adquirir, una Actividad Profesional Confiable (EPA) -por sus siglas en inglés, Entrustable Professional Activity- debe comprobar que el alumno tenga la capacidad de realizar una actividad profesional en la práctica clínica de manera confiable (2). Las competencias describen actividades realizadas por una persona, los estudiantes adquieren competencias, por lo tanto, es una característica del individuo, por ejemplo: adquirir la habilidad de obtener una historia clínica. En contraste, las EPAs son unidades de práctica profesional que se conciben como tareas o responsabilidades, describen una actividad o trabajo, son independientes de la persona, por ejemplo: obtener una historia clínica. Las EPAs descritas inicialmente por ten Cate $(3,4)$ tienen las características siguientes:

- Son actividades realizadas por un profesional en la práctica clínica.

- Pueden ser ejecutadas en un periodo de tiempo.

- Requieren de conocimientos, habilidades y actitudes adquiridas durante el entrenamiento.

- Son observables y medibles, por lo tanto, evaluables.

- Usualmente son realizadas por personal calificado y

- Son independientemente ejecutables.

Adicionalmente, las EPAs son unidades holísticas de trabajo, no solo el conocimiento o la habilidad es importante, sino todo lo que está relacionado para lograr la confiabilidad de una EPA. Cuando un supervisor otorga la confianza a un estudiante no solo reconoce su habilidad, también reconoce su integridad, la confiabilidad de su conducta, su profesionalismo y su humildad, esto es, su capacidad de solicitar ayuda si la requiere (5).

Los niveles, según la confiabilidad a adquirir por el estudiante, son los siguientes:

- Dirigido: La redirección o intervención requerida para realizar la actividad es mayor.

- Soporte: La redirección o intervención requerida es menor.

- Competente: No se necesitó intervenir o la intervención fue mínima.

- Proficiente: Se observó una actuación ejemplar al realizar la actividad.

Revista de Neuro-Psiquiatría. Lima, Perú.

2 Facultad de Medicina Alberto Hurtado, Universidad Peruana Cayetano Heredia. Lima, Perú.

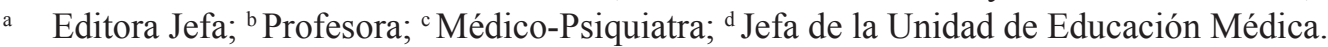


Los últimos dos niveles, competente y proficiente, indican que el alumno es confiable para realizar la actividad sin supervisión. Otra manera de evaluar la confiabilidad de la actividad es según el nivel de supervisión requerida, pudiendo ser: con supervisión directa y el supervisor presente, supervisión indirecta con el supervisor inmediatamente disponible, con la supervisión a distancia, sin supervisión y es capaz de supervisar a otros (6).

Las EPAs pueden organizarse en contextos diversos (5), tales como: programación en servicios generales o en unidades según trastornos, ejecución de procedimientos específicos, actividades no relacionadas al cuidado, por ejemplo, realizar auditorías, evaluar la calidad y seguridad del paciente. Una EPA requiere que el alumno integre múltiples competencias de diferentes dominios para presentar un enfoque integral del paciente (6).

Una de las instituciones líderes en educación médica, como es el Royal College of Physicians and Surgeons of Canada publicó en el 2020 las EPAs para psiquiatría. En el programa se presentan dos EPAs de transición a la disciplina (transition to discipline), cinco EPAs base (foundation) y diez EPAs nucleares (core). Asimismo, se describen las características de cada EPA, el plan de evaluación, que incluye el número de observaciones directas requeridas y los hitos o competencias relevantes (7). Las EPAs presentadas en el documento son las siguientes (tabla 1):

En el 2019 el Consejo Nacional de Residentado Médico (CONAREME) organizó grupos de trabajo de nueve especialidades, incluida psiquiatría, para definir los roles, las competencias claves y competencias habilitadoras

Tabla 1. Actividades profesionales confiables para psiquiatría, 2020 (7).

\section{De transición a la disciplina}

EPA 1: Obtener una historia psiquiátrica para dar una impresión diagnóstica preliminar en pacientes que presentan trastornos mentales.

EPA 2: Comunicar hallazgos clínicos de forma oral y escrita / electrónica

\section{Base}

EPA 1: Evaluar, diagnosticar y participar en el manejo de pacientes con presentaciones médicas relevantes para la psiquiatría

EPA 2: Realizar evaluaciones psiquiátricas con un enfoque biopsicosocial y desarrollar diagnósticos diferenciales básicos para pacientes con trastornos mentales.

EPA 3: Desarrollar e implementar el plan de manejo para pacientes con presentaciones psiquiátricas de complejidad baja a media.

EPA 4: Evaluar el riesgo que presente un plan de seguridad aguda para pacientes que presentan riesgo de daño a sí mismos o a otros.

EPA 5: Realizar una valoración crítica y presentar literatura psiquiátrica.

\section{Nucleares}

EPA 1: Desarrollar planes integrales de tratamiento / manejo para pacientes adultos.

EPA 2: Realizar evaluaciones psiquiátricas y proporcionar diagnósticos diferenciales y planes de manejo para niños y jóvenes.

EPA 3: Realizar evaluaciones psiquiátricas y proporcionar diagnósticos diferenciales y planes de manejo para adultos mayores.

EPA 4: Desarrollar formulaciones biopsicosociales integrales para pacientes a lo largo de la vida,

EPA 5: Identificar, evaluar y gestionar la atención psiquiátrica a lo largo de la vida en situaciones de emergencia.

EPA 6: Integrar los principios y habilidades de la psicoterapia en la atención del paciente.

EPA 7: Integrar los principios y habilidades de la neuroestimulación en la atención del paciente.

EPA 8: Integrar los principios y habilidades de la psicofarmacología en la atención del paciente.

EPA 9: Aplicar la legislación y los principios legales pertinentes a la atención del paciente y de la práctica clínica.

EPA 10: Enseñar a estudiantes, residentes, al público y a otros profesionales de la salud. 
de las especialidades siguiendo el modelo de CanMEDS (8). Este sería un primer paso al estimular un programa por competencias en nuestra especialidad para luego idealmente enfocarlo en EPAs. El definir, en nuestro medio, la educación por competencias con un enfoque basado en EPAs podría ser una estrategia eficaz para garantizar la calidad de los egresados de los diferentes programas de psiquiatría del país.

\section{REFERENCIAS BIBIOGRÁFICAS}

1. Sarabia S. Educación médica basada en competencias. Rev Neuropsiquiatr. 2015; 78(3): 121-122. DOI: 10.20453/rnp.v78i3.2568

2. Soto-Aguilera CA, Robles-Rivera K, Fajardo-Ortiz G, Ortiz-Montalvo A,nHamui-Sutton A. Actividades profesionales confiables (APROC): un enfoque de competencias para el perfil medico. FEM. 2016; 19 (1): 55-62.

3. Ten Cate O, Chen HC, Hoff RG, Petres H, Curriculum development for workplace using entrustable profesional activities (EPAs): AMEE Guide No. 99. Med Teach. 2015;37(11): 983-1002. doi: 10.3109/0142159X.2015.1060308

4. Ten Cate O. A primer on entrustable professional activities. Korean J Med Educ. 2018; 30(1): 1-10.

5. Ten Cate O, Chen C. Entrustable professional activities. Dundee: Association for Medical Education in Europe; 2019. (Citado el 06 de diciembre de 2021). Disponible en: https://www.askamee.org/epa

6. Harden RM, Laidlaw JM. Essential skills for a medical teacher. An Introduction to teaching and learning in medicine. 3rd Edition. Poland: Elsevier Limited; 2021.

7. Royal College of Physicians and Surgeons of Canada. Entrustable professional activities for psychiatry 2020; Version 1.0. Ottawa: Royal College of Physicians and Surgeons of Canada; 2020. (Citado el 12 de diciembre de 2021). Disponible en: https:// www.psychiatry.utoronto.ca/sites/default/files/epaguide-psychiatry-e.pdf

8. Royal College of Physicians and Surgeons of Canada. CanMEDS: Better standards, better physicians, better care. Ottawa: Royal College of Physicians and Surgeons of Canada. (Citado el 12 de diciembre de 2021). Disponible en: https://www.royalcollege. $\mathrm{ca} /$ rcsite/canmeds/canmeds-framework-e 